replicated DNA must prevent the conversion of the M form to active MPF, rather than controlling the conversion of the $\mathrm{S}$ to the $\mathrm{M}$ form. The second possibility is that the conversion of the $S$ to the $M$ form cannot occur until DNA replication has been completed and is itself part of the system of checks ensuring replication is completed before mitosis begins.

Finally, there is the question of how some cells suppress the regular alternation of $\mathrm{S}$ and $\mathrm{M}$ phases. Cells become poly- ploid by allowing many rounds of DNA replication without intervening mitoses. Do these cells act like the diploidizing mutants in $c d c 2$, by degrading $\mathrm{p} 34^{\text {cdc2 }}$, at the end of each round of DNA replication to destroy the $\mathrm{M}$ form, or do they have some other means for inactivating the block to re-replication?

Andrew W. Murray is in the Department of Physiology, University of California, San Francisco, California 94143-0444, USA.

\title{
Glueballs and exotic matter
}

\section{Frank Close}

IF photons - the quanta of electromagnetic radiation - were electrically charged, positive and negatively charged photons could attract and form neutral clusters of light. But such 'photon-balls' do not exist as photons are electrically neutral. However, the quark theory of strongly interacting particles, such as neutrons and protons, implies that there should exist new forms of matter such as 'glueballs' similar in spirit to these imaginary photon-balls. This idea has been around for nearly 20 years and searches for glueballs have been going on almost as long. There are still no unambiguous sightings, yet - as contributions at recent meetings* showed - there is no slackening of interest in the idea.

The structure of bulk matter, molecules and individual atoms involves the underlying attractions and repulsion of positively charged atomic nuclei and their surrounding negatively charged electrons. Disturbing these electromagnetic forces liberates radiation which emerges as quantum bundles - massless photons. The relativistic quantum theory of the electromagnetic field is quantum electrodynamics or QED for short.

Our picture of the more powerful forces at work within the atomic nucleus has been developing for over 50 years. We know that the nucleus's constituent protons and neutrons are not fundamental, but are built from deeper constituents - the quarks. These are electrically charged but also carry a further charge known as colour. Whereas electrical charge comes in positive or negative forms, the amounts measured by simple numbers (a U(1) theory), the quarks' colour charge can have any of three varieties, described by a $3 \times 3$ unitary matrix (an SU(3) theory). To heighten the analogy with QED one can think of the quarks as having positive colour charge, and their antiparticle counterparts - the antiquarks - negative charge. The

* Rutherford-Appleton Laboratory Annual Review Meeting. 17-19 December 1990: Hadron '90. St Goar, Germany, 3-8 September 1990 resulting theory of colour-induced forces is called quantum chromodynamics (QCD) by analogy with QED.

The chromostatic forces - attractions between unlike colours - naturally draw quark to antiquark forming ephemeral states of 'quarkonium' (by analogy with positronium, the electron-positron 'atom'). The psi, upsilon and pion are examples of these states. The threefold colour charge gives further possible attractions among unlike colours. It is possible for three quarks to attract one another so long as each carries a different colour. Thus one naturally finds stable clusters of three quarks, of which the proton and neutron are the best known examples. This simple picture - quark plus antiquark or three-quark clusters accommodates essentially all of the hundreds of short-lived sub-nuclear states discovered in half a century. Yet there is a tantalizing missing ingredient. This is where the glueballs come in.

QCD implies that there will be a colour radiation analogous to electromagnetic radiation; the quantum bundles are known as gluons (contrast the photons). So far it all seems similar to QED. But the presence of three colours for the quarks and the SU(3) ('non-Abelian') structure of QCD implies that the gluons carry colour themselves. Here we see an essentially new structure in QCD, richer in detail than that of QED. The photons of QED are uncharged and travel through space independently; the gluons of QCD are colour-charged and can mutually interact en route. This causes the longrange nature of the forces between quarks (transmitted by the exchange of gluons) to differ from the inverse-square law of QED - a satisfying feature that probably underwrites the close confinement of quarks. But it also implies that bound states of pure glue (the glueballs) can exist.

An obstacle for glueball hunters is that the overall properties of these states seem to be very similar to many conventional quarkonium states, so that their inner constitution is not easily manifested.
Making explicit predictions of their properties from QCD is currently beyond our analytical ability and so theorists have tended to construct tractable models that contain what are thought to be the important features of the dynamics. Although the various models tend to differ in detail, nonetheless they make predictions that agree in several broad features. The lightest glueballs are predicted to occur in the region of $1-2 \mathrm{GeV}$ (one to two times the mass of a proton), to be short lived, and to have total intrinsic angular momentum (spin) of 0 or 2 units, where the proton's spin is half a unit. Their wavefunctions are eigenstates under (effectively unchanged by) spatial reflection (parity); the spin-0 with positive parity $\left(0^{+}\right)$is uniformly predicted to be the lightest; a spin- 0 with negative parity $\left(0^{-}\right)$ or the spin- 2 positive parity $\left(2^{+}\right)$is expected to be the next heaviest.

For several years there have been three particular experimentally observed states with these quantum numbers that are prima facie candidates and fit the predictions but there has been much argument over how sure one can be of describing these as glueballs. Several recent developments add support to the belief that quarks alone are insufficient to describe all of particle spectroscopy. At the meeting Hadron 90, high-quality data from CERN and the US laboratories were announced that greatly elucidated quarkonium spectroscopy in the $1-2 \mathrm{GeV}$ mass region; a result of this is that states are now being identified that do not appear to fit in with the simple quark picture.

Advances in simulating QCD theory on computers where space-time is treated as a collection of discrete points (lattice QCD) were reported at the Rutherford meeting ( $R$. Kenway, Edinburgh University). These computations are now giving encouraging agreement with the masses of known states and predict that the lightest glueballs have $0^{+}$and $2^{+}$quantum numbers, the latter being some 50 per cent more massive than the former. These predictions are tantalizingly akin to data where $2^{+}(1.7 \mathrm{Gev})$ and $0^{+}$(around $1 \mathrm{GeV}$ ) have been favouritc candidates for gluonic states. However, the lattice calculations still suffer from severe approximations - the glueball states are computed with the assumption that no quarks exist.

This also relates to a problem for interpretation of data. It is unrealistic to imagine that physical states will be pure glue; quantum mechanics implies that there will probably be a mixing of character with quarkonium states whose quantum numbers are the same as those of the pure glue states. So the immediate strategy is to try to identify several states, with the same spin and parity quantum numbers, and hopefully find that they are too numerous to fit into a simple quarkonium spectroscopy. News at 
Hadron 90 is that this seems to be the case, at least for the $0^{-}$quantum number ('pseudoscalar states'). This begins to add empirical encouragement to many theorists who have argued (the most recent in a long line being G. J. Gounaris and J. E. Paschalis; Phys. Lett. B251, 634-638; 1990) that detailed structures in spectroscopic data around $1.4 \mathrm{GeV}$ mass are best understood if there is a 0 glueball.

The importance in identifying a detailed spectroscopy of glueballs is that sighting bound states of gauge fields (such as glueballs), as distinct from those of fermions (the conventional 'material' particles), would be entirely new. We have good evidence that the underlying mathematical structure of QCD and QED are very similar but the richness of the SU(3) non-Abelian structure of QCD implies that there are novel possibilities such as non-trivial vacua, instantons and probably further profound consequences that will be revealed only through experiment. To identify these it is important to isolate the spectroscopy of the glueballs. Theory suggests that glueballs are accessible at rather low energies, well within the reach of existing laboratories. It will be the advances in detector technology and particle beam quality and intensity that will be the keys to opening up this area of particle physics, and will offer the hope of deepening insights into the physics of nonAbelian gauge field theories.

Frank Close is at the Rutherford-Appleton Laboratories, Chilton, Didcot $\mathrm{OX} 11 \mathrm{OQH}$, UK, and Oak Ridge National Laboratory, PO Box 2008, Oak Ridge, Tennessee 37831, USA.

\section{Real and imagined dangers}

\section{Gordon Ada}

EARLIER this month a report by Oehen et al. appeared in Science ${ }^{1}$, under the title "Vaccination for Disease", in which the authors describe some experiments with lymphocytic choriomeningitis virus (LCMV) in mice. This virus contains two main protein types, glycoproteins and a nucleoprotein. In some experiments, preimmunization of mice with vaccinia virusLCMV single protein constructs exacerbated the disease that occurred when the mice were challenged with LCMV administered intracerebrally. In contrast, preimmunization with intact LCMV protected the mice. Although these experiments are valid and helpful lessons can be drawn from them, the very title of the paper might be inappropriately taken as a cause for alarm, particularly as chimaeric viruses (or bacteria) have already been successfully used for vaccination.

Elsewhere, I have proposed ${ }^{2}$ that a vaccine should meet four immunological requirements, three of which are relevant here. One is that the vaccine should contain a sufficient number of different T-cell epitopes that $\mathrm{T}$-cell responses will be achieved in all members of a genetically diverse outbred population. Inclusion of several different proteins in the preparation increases the number of available epitopes. A second requirement is that the vaccine should generate a large pool of memory $\mathrm{T}$ (and $\mathrm{B}$ ) cells; the greater the size of this T-cell pool, the quicker the appearance of effector $\mathrm{T}$-cell activity generated from the memory cells by the challenge virus. The results of Oehen et al. are consistent with these points, as shown in their Figs 1 and $2 \mathrm{C}$. As expected, intact LCMV which contains all the viral antigens was more protective than either of the chimaeric vaccinia virus constructs.
A third requirement of a vaccine is the generation and persistence of high titres of neutralizing antibody so that the great bulk of a challenge virus, at any time after immunization, is prevented from infecting susceptible cells. Oehen et al. did not measure such antibody generated by any of the immunization schedules, but it is very likely that immunization with whole virus would have achieved this most effectively. This, as well as the rapid appearance of effector $T$ cells after challenge (their Fig. 2C), may have been a deciding factor in preventing the deaths of animals that had been preimmunized with intact virus.

Several, more general, points should also be made. First, it is inevitable that $T$-cell activation will result in some immunopathological effects. Interleukins, which are defined as mediators of inflammation, are secreted by both $\mathrm{CD}^{+}$and $\mathrm{CD}^{+}$cells. The trick is to limit the effect as far as possible and this can be done by minimizing the extent of infection that occurs upon challenge. The risk of immunopathology becomes greater when, for reasons such as great antigenic variation, the virus largely escapes the neutralization of infectivity by preformed antibody.

Second, LCMV infection is an unusual model. Challenge by intracerebral injection, used in the experiments of Oehen $e t$ $a l$, is an unnatural route of infection. Virus inoculated into the brain can form a reservoir not easily available to the immune system. Other than vector-borne agents, most agents infect through a mucosal surface and there is increasing interest in developing vaccines which will stimulate a secretory $\operatorname{Ig} \mathrm{A}$ response as this is the first line of defence.

Third, there are many examples with other viruses where transfer of virusspecific cytotoxic $T$ cells before or after administration of a lethal dose of virus has cleared the infection and prevented death. In addition, there are many examples in model systems where immunization with a chimaeric virus preparation has provided protection against a challenge dose of the infectious agent which was the source of the foreign antigen $(\mathrm{s})^{3}$. Two outstanding examples are vaccinia virus constructs which contain antigen from rabies virus ${ }^{\text {t.5 }}$ or from rinderpest virus ${ }^{6}$ - foxes immunized with the vaccinia-rabies virus construct survived challenge with many thousands of lethal doses of rabies virus; cattle immunized with the vacciniarinderpest virus construct were protected against challenge by a thousand lethal doses of rinderpest virus.

Most vaccines currently in use are to control acute infections. Among the most successful are those consisting of live, attenuated viruses which induce cytoxic T-cell responses. Many of the infectious agents that are the target for vaccine development cause chronic persisting infections. The observations of Oehen et al. may be relevant in some of these cases, for example in HIV infections where there is great antigenic variation in the envelope protein. A candidate vaccine now in phase 1 human trials is a construct composed of vaccinia virus and the HIV envelope protein. The rationale behind this approach is to generate cytotoxic $\mathrm{T}$-cell responses which would limit infection caused by either free virus or infected cells. It remains highly desirable that such a vaccine should still aim to induce cross-protective neutralizing antibodies to limit the extent of viral infection upon initial exposure, and this may now be possible? The current development of a vaccinia virus construct containing three HIV antigens is a useful further approach to achieve rapid elimination of infected cells.

Although it may never be possible to guarantee that a given approach will always result in a completely safe vaccine, enough is now known about the important immunological requirements for vaccines, especially for viral infections, to proceed with confidence in developing the next generation. The use of live vectors, viruses or bacteria, is one of several approaches in use; it may turn out to be the best in some situations.

Gordon Ada is in the School of Hygiene and Public Health. The Johns Hopkins University, Baltimore, Maryland 21205, USA.

\footnotetext{
1. Oehen, S., Hengartner, H. \& Zinkernagel, R.M. Science 251, 195-198 (1991)

2. Ada, G. L. Lancet 335, 523-526 (1990)

3. Flexner, C. \& Moss, B. in New Generation Vaccines (eds Woodrow, G.C. \& Levine, M.M.) 189-206 (Dekker, New Woodrow, G.

4. Blancou, F. et al. Nature 332, 373-375 (1986)

5. Brochien, B. et al. Vaccine 8, 101-104 (1989)

6. Yilma, T.D. et al. Science 242, 1058-1061 (1988)

7. Javaherian, K. et al. Science 250, 1590-1593 (1990)
} 